\title{
Diseño, construcción y simulación de la automatización de una máquina selectora de tuercas según su tamaño
}

\author{
Design, Construction and Simulation of a Machine that sorts nuts by their size
}

\author{
Oscar Ferney Villada Molina, Omar Andrés Hincapié Marín, Francisco Javier Henao Castañeda \\ Facultad Ingeniería Mecánica, Universidad Tecnológica, Pereira, Colombia \\ osfervi89@hotmail.com, oahincapiedutp.edu.co, frajahec@utp.edu.co
}

\begin{abstract}
Resumen- El presente trabajo tiene como objetivo el diseño y construcción de una máquina clasificadora de tuercas, centrándose principalmente en la separación de acuerdo a su tamaño, empleando un sistema de clasificación por medio de planos inclinados y aplicando principios básicos de vibraciones mecánicas junto con la fuerza de la gravedad. Teniendo en cuenta, algunos parámetros previamente establecidos tales como ángulo de inclinación, separación entre planos y la frecuencia de vibración.
\end{abstract}

Palabras clave - ángulo, construir, clasificar, diseñar, frecuencia, ordenar, separar, simular, vibración.

Abstract - This work treats about design and construction of a nuts sorting machine that basically separates them according to their sizes, using bent plates and applying mechanical vibration principles along with the gravity force. Some parameters like bend angle, plates separation and vibrations frequency are taken into account too.

Key Word — angle, build, sort, design, frequency, sorting, separating, simulate vibration.

\section{INTRODUCCIÓN}

Los sistemas de clasificación que nacen a partir de la implementación de elementos mecánicos, son desarrollados debido a la necesidad que tienen las empresas dedicadas a la producción en masa no solo de piezas o elementos mecánicos sino también agroindustriales, de realizar un proceso de selección sobre la producción con el fin de separar y tener un mejor control de sus productos de una manera más lógica y ordenada, aplicando una serie de criterios en la distinción de ellos para reconocer y apartar de manera oportuna los artículos que simplemente no cumplen con las especificaciones o normas mínimas requeridas en dichas piezas para su elaboración.

Teniendo como objetivo el diseño y construcción de una máquina clasificadora de tuercas se realiza una investigación previa partiendo de las normativas, teorías y tecnologías aplicables y útiles dirigiendo el proyecto hacia la búsqueda de una solución simple y puntual a una necesidad en la industria. El alcance del proyecto consiste en diseñar y construir un sistema de clasificación, cuya aplicación pueda ser implementada de manera general a una gran cantidad de procesos con características similares a las planteadas en este documento.

\section{CONTENIDO}

\section{A. Diseño}

Según C.L. Dym en "A.I Aproaches to Engineering design: Taxonomies and search prescription, 1990" define el diseño de ingeniería en la forma siguiente:

"El diseño de ingeniería es la generación y evaluación sistemática e inteligente de las especificaciones para los artefactos cuya forma y funcionamiento alcanzan los objetivos planteados y satisfacen las restricciones especificadas."[12].

\section{B. Automatización}

La palabra automatización, proviene del griego antiguo auto: guiado por uno mismo.

La automatización como una disciplina de la ingeniería, es más amplia que un mero sistema de control, abarca la instrumentación industrial que incluye los sensores y transmisores de campo, los sistemas de control y supervisión, los sistemas de transmisión y recolección de datos y las 
aplicaciones de software en tiempo real para supervisar $\mathrm{y} \mathrm{F}_{1}=\mathrm{k}_{1} * \mathrm{x}, \mathrm{F}_{2}=\mathrm{k}_{2} * \mathrm{x}$

controlar las operaciones de plantas o procesos industriales.

Esto se lleva a cabo mediante el siguiente conjunto de técnicas: diseño (con ayuda del computador CAD), simulación (el diseño aplicado a la ingeniería CAE) y fabricación (desarrollo del producto aplicando el diseño y la ingeniería CAM [3].

\section{Definición de vibración}

$\mathrm{F}_{1}=\mathrm{F}_{2}=\frac{\mathrm{F}}{2}$

$\mathrm{k}_{1}=\frac{\mathrm{F}}{2 \mathrm{x}}$

$\mathrm{k}_{1}=\frac{\mathrm{m} * \mathrm{~g}}{2 *\left(\mathrm{x}_{\mathrm{i}}-\mathrm{x}_{\mathrm{f}}\right)}$

La vibración es toda variación en el tiempo, de una magnitud que describe el movimiento o la posición de un sistema mecánico, cuando esta magnitud es alternativamente mayor o menor que cierto valor promedio de referencia [14].

D. Desarrollo del diseño y construcción

1. Elaboración de cálculos de diseño

$\mathrm{k}_{1}=\frac{186.39 \mathrm{~N}}{2 *(0.05-0.045) \mathrm{m}}$

$\mathrm{k}_{1}=\mathrm{k}_{2}=18639 \mathrm{~N} / \mathrm{m}$

2. Fórmulas más relevantes empleadas para el modelo de vibración.

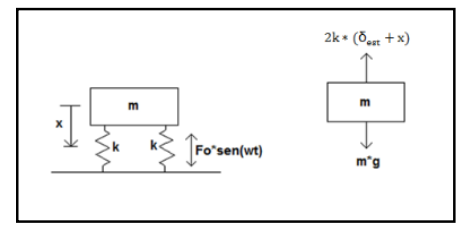

$\mathrm{x}$; es un desplazamiento uniforme

$\mathrm{F}_{1}+\mathrm{F}_{2}=\mathrm{F}_{\mathrm{T}}$

$\mathrm{k}_{\mathrm{eq}}=\frac{\mathrm{F}_{\mathrm{T}}}{\mathrm{x}}$

$\mathrm{k}_{\mathrm{eq}}=\frac{\left(\mathrm{k}_{1}+\mathrm{k}_{2}\right) * \mathrm{x}}{\mathrm{x}}$

$\mathrm{k}_{\mathrm{eq}}=\left(\mathrm{k}_{1}+\mathrm{k}_{2}\right)$

$\mathrm{k}_{\mathrm{eq}}=37278 \mathrm{~N} / \mathrm{m}$

Figura 1. Diagrama de cuerpo libre sistema equivalente de la máquina.

Datos conocidos:

$\mathrm{m}=19 \mathrm{~kg}$

$\mathrm{g}=9.81 \mathrm{~m} / \mathrm{s}^{2}$

$x_{i}=5 \mathrm{~cm}$

$\mathrm{x}_{\mathrm{f}}=4.5 \mathrm{~cm}$

Empleando la ley de movimiento de newton:

$\sum \mathrm{m} * \ddot{\mathrm{x}}=($ sumatoria de fuerzas en la direccion $\mathrm{x})$

$\sum \mathrm{m} * \ddot{\mathrm{x}}=-2 \mathrm{k}\left(\mathrm{x}+\delta_{\text {est }}\right)+\mathrm{mg}-\mathrm{c \dot {x }}+\mathrm{F}_{0} \operatorname{sen}(\omega * \mathrm{t})$

Pero $2 \mathrm{k} \delta_{\text {est }}=\mathrm{mg}$ donde $\mathrm{mg}$ es el peso de la masa por tanto, la ecuación de movimiento toma su forma más general.

$(\mathrm{m} * \ddot{\mathrm{x}})+\mathrm{cx}+2 \mathrm{kx}=\mathrm{F}_{0} \operatorname{sen}(\omega * \mathrm{t})$

Como el sistema no tiene amortiguación se simplifica aún más la ecuación.

$(\mathrm{m} * \ddot{\mathrm{x}})+2 \mathrm{kx}=\mathrm{F}_{0} \operatorname{sen}(\omega * \mathrm{t})$

Hallando el resorte equivalente para este sistema se tiene.

3. Fórmulas más relevantes empleadas para el diseño por fatiga.

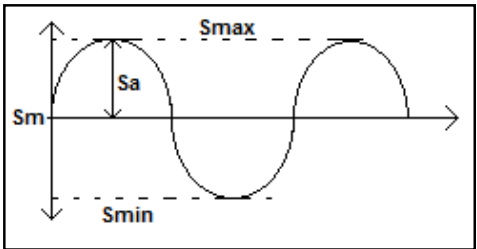

Figura 2. Diagrama de esfuerzos de la máquina

a. Teoría de fatiga

$\frac{1}{\mathrm{~N}}=\frac{\mathrm{k}_{\mathrm{fm}} * \mathrm{~S}_{\mathrm{ms}}}{\mathrm{S}_{\mathrm{us}}}+\frac{\mathrm{k}_{\mathrm{ff}} * \mathrm{~S}_{\mathrm{as}}}{\mathrm{S}_{\mathrm{n}}} ; \mathrm{Si} \quad \mathrm{S}_{\mathrm{ms}}+\mathrm{S}_{\mathrm{as}}<\frac{\mathrm{S}_{\mathrm{ys}}}{\mathrm{N}}$

De lo contrario se emplearía.

$\frac{1}{\mathrm{~N}}=\frac{\mathrm{S}_{\mathrm{ms}}+\mathrm{S}_{\mathrm{as}}}{\mathrm{S}_{\mathrm{ys}}}$

$S_{m}=\frac{S_{\max }+S_{\min }}{2} ;(+0-)$

$S_{a}=\frac{S_{\max }-S_{\min }}{2} \quad ;\left(S_{a} \geq 0\right)$

$k_{a}=$ Coeficiente superficial 
$k_{b}=$ Coeficiente de tamaño

$k_{c}=$ Coeficiente de confiabilidad

$k_{d}=$ Coeficiente de temperatura

$k_{e}=$ Coeficiente de efectos varios

$k_{c a r}=$ Coeficiente de carga

$k_{f} ; k_{f m} ; k_{f f}=$ Factores de concentración de esfuerzos

$k_{f}=1+q *\left(k_{t}-1\right)$

Índice de sensibilidad a la entalla

$q=\frac{1}{1+\frac{\sqrt{a}}{\sqrt{r}}}$ Donde q está entre $(0 \leq q \leq 1)$ y $\sqrt{ } a$ es la constante de Neuber.

Datos del material:

Acero 1020 laminado en frio.

$S_{y}=207 M p a$

$S_{u}=379 \mathrm{Mpa}$

$\% e=15 \%$

$H B=111$

$m_{2}=1.4 \mathrm{~kg}$

$\Phi_{i}=12.7 \mathrm{~mm}=\frac{1}{2} \mathrm{in}$

$\Phi_{0}=15.875 \mathrm{~mm}=\frac{5}{8} \mathrm{in}$

$C=R=7.9375 \mathrm{~mm}=\frac{5}{16} \mathrm{in}$

Asumiendo que la sección crítica está sometida al peso máximo que ejerce el plano en una de sus guías.

$\mathrm{F}=\mathrm{m}_{2} * \mathrm{~g} \approx 1.4 \mathrm{~kg} * 9.81 \frac{\mathrm{m}}{\mathrm{s}^{2}}=13.734 \mathrm{~N}$

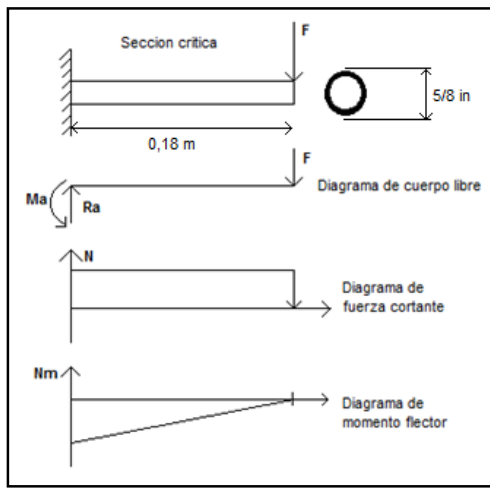

Figura 3. Diagrama de cuerpo libre elemento más crítico de la máquina

$\mathrm{F}_{\mathrm{a}}=\mathrm{F}_{\mathrm{m}}=\frac{13.734 \mathrm{~N}}{2}=6.867 \mathrm{~N}$

$\mathrm{S}_{\mathrm{a}}=\mathrm{S}_{\mathrm{m}}=\mathrm{MC} / \mathrm{I}$

$\mathrm{M}_{\mathrm{m}}=\mathrm{M}_{\mathrm{a}}=6.867 \mathrm{~N} * 180 \mathrm{~mm}=1236.06 \mathrm{~N} * \mathrm{~mm}$

$\mathrm{I}=\frac{\pi}{64}\left(\Phi_{0}^{4}-\Phi_{1}^{4}\right)=1840.64 \mathrm{~mm}^{4}$

$\mathrm{S}_{\mathrm{a}}=\mathrm{S}_{\mathrm{m}}=5.33 \mathrm{MPa}$

$\mathrm{k}_{\mathrm{a}}=\mathrm{a} \times \mathrm{S}_{\mathrm{u}}^{\mathrm{b}}=0.9350$

$\mathrm{k}_{\mathrm{b}}=1$

$\mathrm{k}_{\mathrm{c}}=0.702$

$\mathrm{k}_{\mathrm{d}}=1$

$\mathrm{k}_{\mathrm{e}}=1 ; \quad($ Para este caso se asume 1$)$

$k_{c a r}=1$

$k_{f}=1+q *\left(k_{t}-1\right)=1 ;$ No existen discontinuidades en el elemento

$k_{f}=k_{f m}=k_{f f}=1 ;$ Para este caso tomaremos $k_{f}=$

$k_{f m}=k_{f f}$

$S_{n}=k * S_{e^{\prime}} ;$ Solo se aplica para vida infinita $n_{c}>10^{6}$

$S_{e^{\prime}}=0.5 * S_{u}=0.5 * 379 \mathrm{MPa}=189.5 \mathrm{MPa}$

$k=k_{b} * k_{c} * k_{d} * k_{e} * k_{c a r} * k_{a}$

$k=0.6563$

$S_{n}=0.6563 * 189.5 \mathrm{MPa}=124.3688 \mathrm{MPa}$ 
De acuerdo a lo anterior la ecuación se expresa de manera más simplificada.

$\frac{1}{N}=\frac{S_{m}}{S_{u}}+\frac{S_{a}}{S_{n}}=\frac{5.33 M P a}{379 M P a}+\frac{5.33 M P a}{124.3688 M P a}=0.05691$

$N=17.56$

\section{E. Selección materiales}

Los materiales seleccionados para la construcción de la máquina selectora de tuercas se basan en la resistencia máxima que soporta el dispositivo calculado previamente, arrojando como resultado la resistencia de fluencia de un acero 1020 que cumple con los límites de resistencia para dicha máquina.

\section{F. Fuente vibración}

Para generar la vibración que es necesaria en el proceso, se recurrió a una fuente de vibración utilizada en el mercado como un masajeador de la marca Oster; el cual trabaja entre $110-120 \mathrm{~V}$ a una potencia de 45 watts

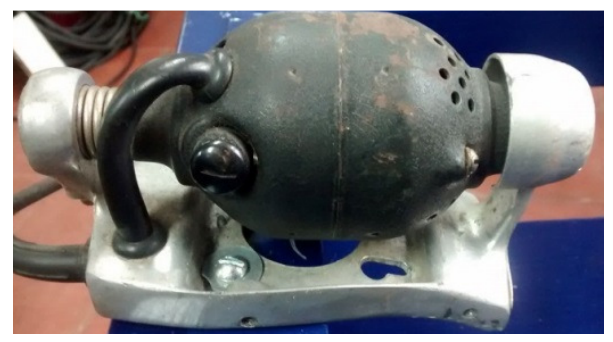

Figura 4. Vibrador mecánico

G. Elaboración de un diseño mediante la utilización de un software (SOLIDWORKS 2012).

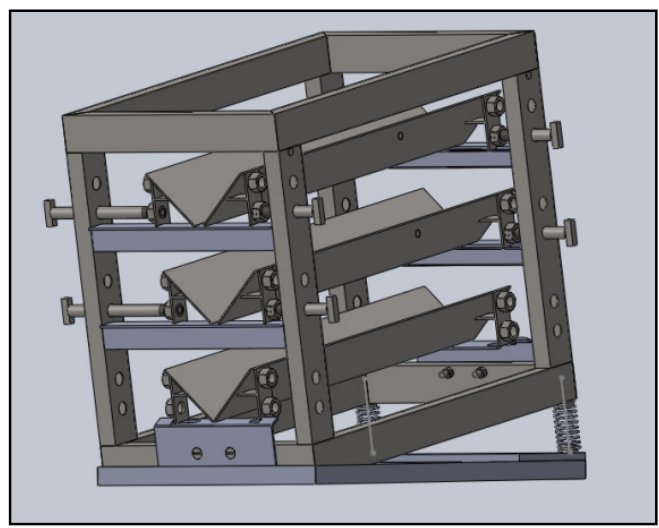

Figura 5. Boceto máquina clasificadora de tuercas

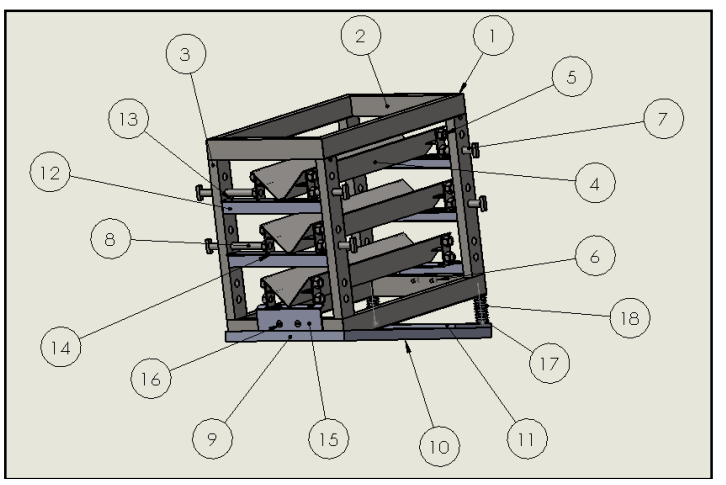

Figura 6. Ubicación de las piezas máquina clasificadora de tuercas

\begin{tabular}{|c|c|c|c|c|}
\hline $\begin{array}{c}\text { N.DE } \\
\text { ELEMENTO }\end{array}$ & N. ${ }^{\circ}$ DE PIEZA & MATERIAL & CANTIDAD & DESCRIPCIÓN \\
\hline 1 & Horizontal Largo 2 & Acero 1020 & 4 & $\begin{array}{l}\text { Tubo Cuadrado } \\
\text { Calibre } 16\end{array}$ \\
\hline 2 & Horizontal Corto 2 & Acero 1020 & 4 & $\begin{array}{l}\text { Tubo Cuadrado } \\
\text { Calibre } 16\end{array}$ \\
\hline 3 & $\begin{array}{l}\text { Verticales } \\
\text { Agujerados }\end{array}$ & Acero 1020 & 4 & $\begin{array}{l}\text { Tubo Cuadrado } \\
\text { Calibre } 16\end{array}$ \\
\hline 4 & Placas & Acero 1020 & 6 & Lamina Calibre 13 \\
\hline 5 & HNUT $0.625-18-\mathrm{D}-\mathrm{N}$ & Acero & 24 & \\
\hline 6 & HNUT $0.3125-18-\mathrm{D}-\mathrm{N}$ & Acero & 4 & \\
\hline 7 & Guia inferior & Acero 1020 & 4 & Acero Calibre 18 \\
\hline 8 & Guia superior & Acero 1020 & 4 & Acero Calibre 18 \\
\hline 9 & $\begin{array}{l}\text { Angulo para base } \\
\text { nueva }\end{array}$ & Acero 1020 & 2 & $\begin{array}{l}\text { Angulo de alas } \\
\text { iguales } 11 / 2^{*} 11 / 2\end{array}$ \\
\hline 10 & $\begin{array}{l}\text { Angulo para base } \\
\text { nueva } 2\end{array}$ & Acero 1020 & 2 & $\begin{array}{l}\text { Angulo de alas } \\
\text { iguales } 11 / 2^{*} 11 / 2\end{array}$ \\
\hline 11 & $\begin{array}{l}\text { Placa para soportar } \\
\text { resortes }\end{array}$ & Acero 1020 & 1 & Lamina Calibre 20 \\
\hline 12 & $\begin{array}{l}\text { Angulo soporta- } \\
\text { placas }\end{array}$ & Acero 1020 & 4 & $\begin{array}{l}\text { Angulo alas } \\
\text { iguales } 2 * 2\end{array}$ \\
\hline 13 & Regleta 1 & Acero 1020 & 2 & Lamina Calibre 13 \\
\hline 74 & Regleta 2 & Acero 1020 & 2 & Lamina Calibre 13 \\
\hline 15 & Apoyo placa inferior & Acero 1020 & 2 & $\begin{array}{l}\text { Angulo alas } \\
\text { iguales } 3^{* 3}\end{array}$ \\
\hline 16 & $\begin{array}{l}\text { CSBOLTO.STI25- } \\
18 \times 2 \times 2-S-N\end{array}$ & Acero & 4 & \\
\hline 17 & Pin Resorte & Acero 1020 & 4 & $\begin{array}{l}\text { Tubo redondo } \\
\text { Calibre } 14\end{array}$ \\
\hline 18 & Resorte & $\begin{array}{c}\text { Acero SAE } \\
5160\end{array}$ & 2 & \\
\hline
\end{tabular}

Tabla 1. Listado de partes máquina clasificadora de tuercas

En la figura 6 se muestra el diseño final de la máquina selectora de tuercas por tamaño, y en la tabla 1 se describe cada uno de los elementos que la componen.

\section{H. Construcción del prototipo.}

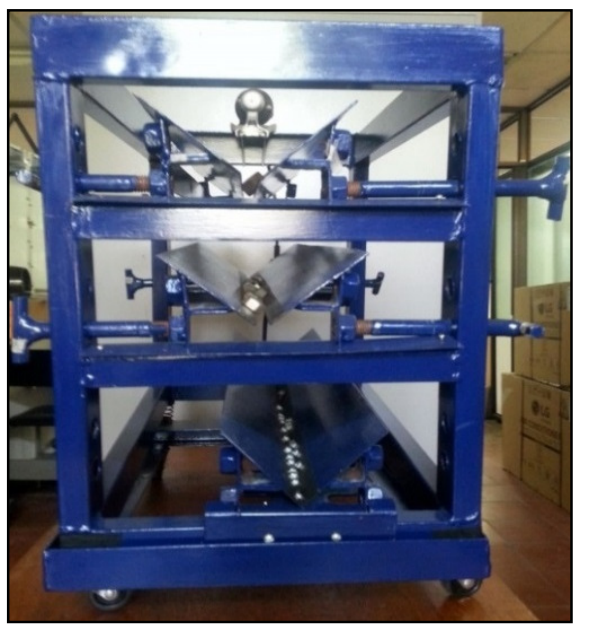

Figura 7. Vista frontal de la máquina selectora de tuercas. 
En la figura 7 se muestra la máquina con las placas en donde se clasifican las tuercas, la separación entre cada una de ellas y la ubicación de la fuente de vibración.

\subsection{Simulación.}

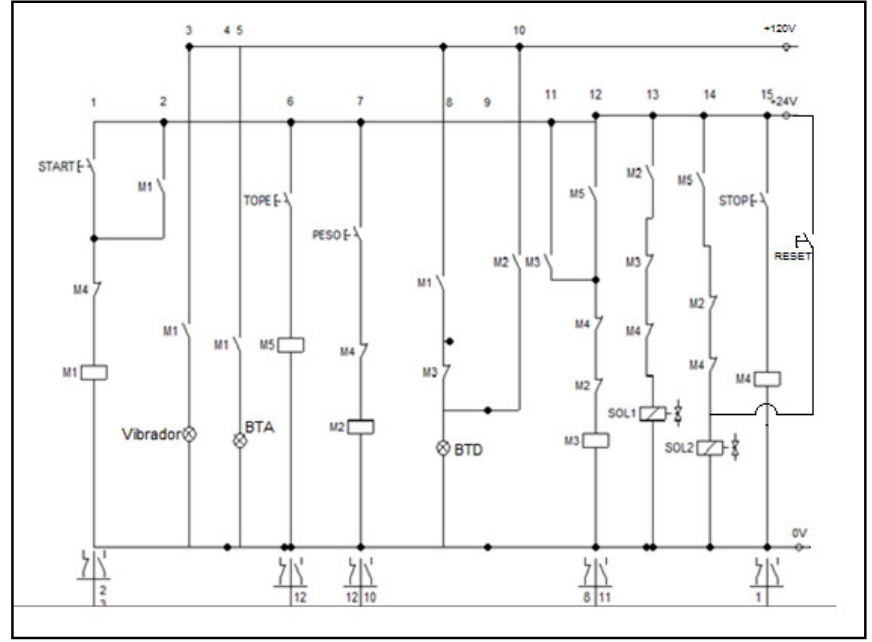

Figura 8. Diagrama Ladder, simulación máquina selectora.

\begin{tabular}{|c|l|l|}
\hline $\mathbf{N}^{\circ}$ & Elemento & \multicolumn{1}{|c|}{ Nombre } \\
\hline 1 & START & Interruptor de encendido \\
\hline 2 & Vibrador & Vibrador \\
\hline 3 & BTA & Dispositivo de alimentacion de tuercas \\
\hline 4 & TOPE & Sensor de contacto \\
\hline 5 & PESO & Sensor de contacto \\
\hline 6 & BTD & Dispositivo de descarga de tuercas \\
\hline 7 & SOL1 & Solenoide 1 \\
\hline 8 & SOL2 & Solenoide 2 \\
\hline 9 & STOP & Interruptor de Apagado y emergencia \\
\hline 10 & M1 & Rele 1 \\
\hline 11 & M2 & Rele 2 \\
\hline 12 & M3 & Rele 3 \\
\hline 13 & M4 & Rele 4 \\
\hline 14 & M5 & Rele 5 \\
\hline 15 & RESET & Interruptor de reinicio \\
\hline
\end{tabular}

Tabla 2. Listado elementos diagrama Ladder.

En la figura 8 (Diagrama Ladder, simulación automatización de maquina selectora de tuercas) se tiene un interruptor de arranque (START) que, al pulsarlo, envía una señal eléctrica que queda almacenada en una memoria $M$. Esta señal energiza el sistema y a su vez da el arranque al vibrador y a la alimentación (BTA) de tuercas.

Las tuercas se clasifican de acuerdo a su tamaño y caen en una serie de recipientes que tienen en la parte inferior unos resortes que se comprimen por el peso que ejerce cada una de las tuercas, hasta llegar al límite establecido para que se active el sensor de contacto (PESO) mandando una señal a la memoria (M2) que activa el solenoide 1, haciendo girar el actuador $90^{\circ}$ y de esta manera las tuercas son entregadas a la siguiente estación de trabajo.

Después de que las tuercas pasan a la estación de trabajo, el actuador llega a un final de carrera (TOPE), que activa el solenoide 2, el cual hace regresar el actuador de giro a su posición inicial siendo esto un proceso continuo (ciclo).

Dado el caso de presentarse un problema en el proceso, se tiene un botón de emergencia (STOP) cuya función es parar la tarea que se estaba ejecutando. Luego de resolver el inconveniente se debe llevar el sistema a sus condiciones iniciales por medio de un pulsador (RESET).

\section{RESULTADOS.}

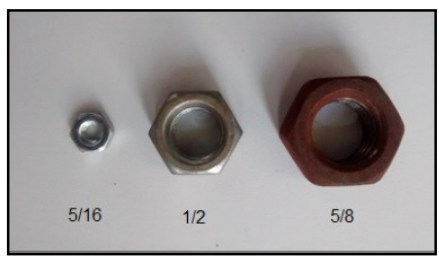

Figura 9. Tuercas utilizadas en las pruebas.

A. Inclinación a un ángulo de $10^{\circ}$

\begin{tabular}{|c|c|c|c|}
\hline \multicolumn{4}{|c|}{ Angulo de Inclinación de 10 } \\
\hline $\mathbf{N}^{\circ}$ & $\begin{array}{c}\text { t. Tuerca } \\
\text { grande [s] }\end{array}$ & $\begin{array}{c}\text { t. Tuerca } \\
\text { Mediana [s] }\end{array}$ & $\begin{array}{c}\text { t. Tuerca } \\
\text { Pequeña [s] }\end{array}$ \\
\hline $\mathbf{1}$ & 60,95 & 117,16 & 61,87 \\
\hline $\mathbf{2}$ & 40,42 & 131,51 & 51,74 \\
\hline $\mathbf{3}$ & 43,88 & 128,66 & 40,85 \\
\hline $\mathbf{4}$ & 45,47 & 146,3 & 47,01 \\
\hline $\mathbf{5}$ & 39,57 & 154,34 & 30,31 \\
\hline Promedio & 46,058 & 135,594 & 46,356 \\
\hline \multicolumn{4}{|c}{} \\
\hline
\end{tabular}

Tabla 3. Datos ángulo de inclinación de $10^{\circ}$

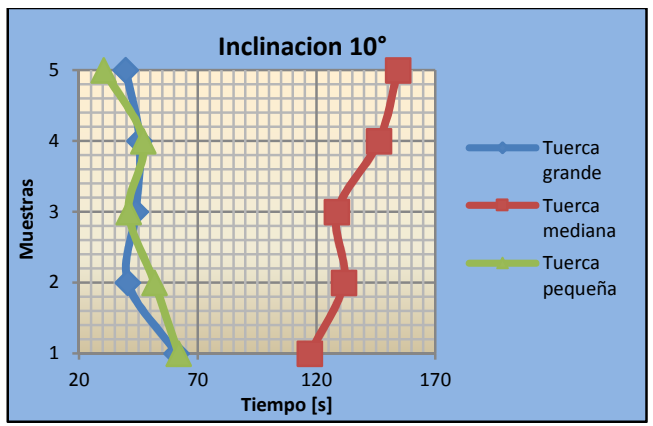

Figura 10. Gráfica datos inclinación de $10^{\circ}$ 
B. Inclinación a un ángulo de $15^{\circ}$

\begin{tabular}{|c|c|c|c|}
\hline \multicolumn{4}{|c|}{ Angulo de Inclinación de 15 } \\
\hline $\mathbf{N}^{\circ}$ & $\begin{array}{c}\text { t. Tuerca } \\
\text { grande [s] }\end{array}$ & $\begin{array}{c}\text { t. Tuerca } \\
\text { Mediana [s] }\end{array}$ & $\begin{array}{c}\text { t. Tuerca } \\
\text { Pequeña [s] }\end{array}$ \\
\hline $\mathbf{1}$ & 18,24 & 73,1 & 23,9 \\
\hline $\mathbf{2}$ & 21,19 & 75,42 & 16,88 \\
\hline $\mathbf{3}$ & 21,32 & 63,42 & 17,99 \\
\hline $\mathbf{4}$ & 18,08 & 64,44 & 18,88 \\
\hline $\mathbf{5}$ & 24,12 & 64,73 & 23,34 \\
\hline Promedio & 20,59 & 68,222 & 20,198 \\
\hline
\end{tabular}

Tabla 4. Datos ángulo de inclinación de $15^{\circ}$

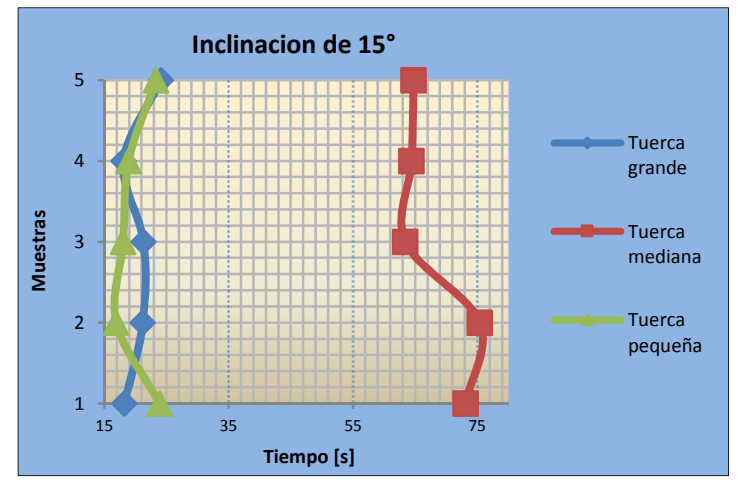

Figura 11. Gráfica datos inclinación de $15^{\circ}$

C. Inclinación a un ángulo de $20^{\circ}$

\begin{tabular}{|c|c|c|c|}
\hline \multicolumn{4}{|c|}{ Angulo de Inclinación de 20 } \\
\hline $\mathbf{N}^{\circ}$ & $\begin{array}{c}\text { t. Tuerca } \\
\text { grande [s] }\end{array}$ & $\begin{array}{c}\text { t. Tuerca } \\
\text { Mediana [s] }\end{array}$ & $\begin{array}{c}\text { t. Tuerca } \\
\text { Pequeña [s] }\end{array}$ \\
\hline $\mathbf{1}$ & 16,98 & 42,26 & 6,51 \\
\hline $\mathbf{2}$ & 15,11 & 35,81 & 11,24 \\
\hline $\mathbf{3}$ & 16,57 & 42,38 & 11,11 \\
\hline $\mathbf{4}$ & 15,5 & 40,42 & 13,61 \\
\hline $\mathbf{5}$ & 15,12 & 40,56 & 15,55 \\
\hline Promedio & 15,856 & 40,286 & 11,604 \\
\hline
\end{tabular}

Tabla 5. Datos ángulo de inclinación de $20^{\circ}$

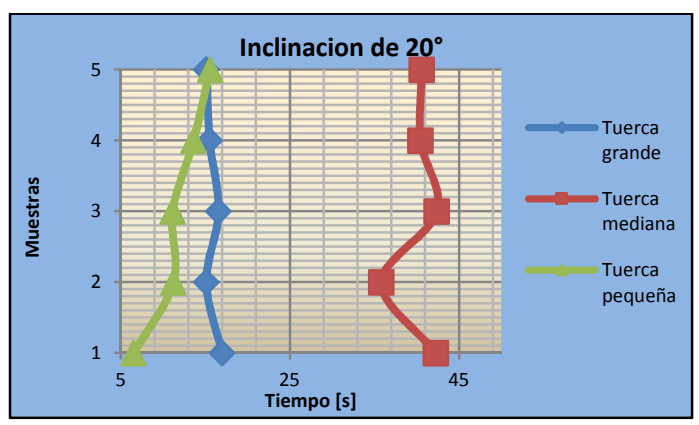

Figura 12. Gráfica datos inclinación de $20^{\circ}$

\section{ANÁLISIS DE RESULTADOS.}

Como se observa en las figuras 10, 11 y 12 hay un patrón de comportamiento que se repite, tanto para las tuercas grandes como para las pequeñas, por lo cual, se tienen tiempos muy similares. Al contrario, la tuerca de tamaño mediano ubicada en el plano medio, se halla más alejada de los tiempos de los otros dos tamaños. Todo esto es a causa del fenómeno ondulatorio generado por el vibrador (figura 13), donde los dos tiempos mayores se dan en el plano superior e inferior; al hacer una analogía es donde se tiene la mayor amplitud. Como resultado, la tuerca tendrá un mayor movimiento o salto, por lo que avanzaría más rápido y los tiempos obtenidos resultan menores con respecto al plano medio.

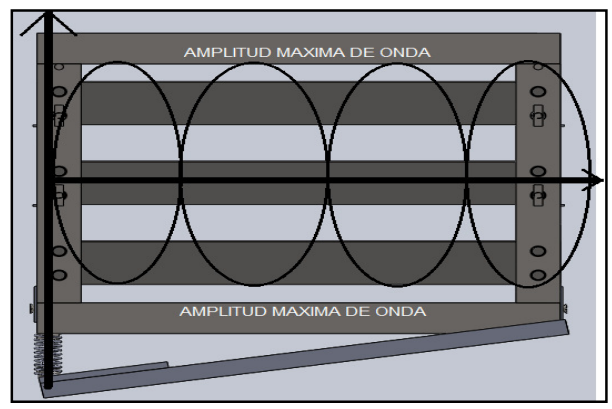

Figura 13. Comportamiento ondulatorio en la máquina.

Al ejecutar las pruebas variando el ángulo de inclinación, se encuentra que para la selección de los diferentes tamaños de tuerca, el menor tiempo se presenta para un ángulo de $20^{\circ}$, como se muestra en la tabla 5 , pero en contraste al tiempo de clasificación no se está llevando de forma correcta la separación de las tuercas.

Cuando se emplea una configuración a un ángulo de $10^{\circ}$ (tabla 3), el tiempo que se tarda la máquina para clasificar las tuercas, es mayor en comparación con las otras dos disposiciones. La forma de clasificación que se encontró más adecuada es a una inclinación de $15^{\circ}$ (tabla 4), donde las tuercas se ubican en sus planos respectivos en un menor tiempo comparado con la configuración a $10^{\circ}$ (tabla 3 ).

\section{CONCLUSIONES}

De acuerdo a la revisión bibliográfica no se encontraron documentos y normativas que pudieran reglamentar el diseño y construcción de una máquina selectora de tuercas según su tamaño, por lo tanto, se recurre a trabajar con el material recolectado y los conocimientos adquiridos durante la carrera de Ingeniería Mecánica.

El movimiento de las tuercas sobre los planos inclinados es proporcional a la frecuencia de vibración aplicada en el 
dispositivo, es decir, cuanto mayor es la vibración de la máquina, la velocidad de las tuercas se incrementa, implicando que sea ésta una de las variables más relevantes en el funcionamiento apropiado.

Al aumentar el ángulo de inclinación de la máquina clasificadora de tuercas, se logra una mayor rapidez entre la entrada y salida de las tuercas a lo largo de los planos, pero menor eficiencia en la clasificación. El ángulo de mejor operación es de $15^{\circ}$ con respecto a la horizontal, debido a que presenta mayor eficiencia en la clasificación, con el menor tiempo.

Al desarrollar las pruebas de funcionamiento, surgió la necesidad de cambiar la fuente de vibración por motivos de diseño y ensamble. La disposición inicial del elemento vibrador no permitía una adecuada clasificación de las tuercas debido a su pequeña capacidad de vibración. De esta manera, se reemplaza este dispositivo por otro de mayor capacidad corrigiendo el problema inicial de clasificación.

\section{RECOMENDACIONES}

Las personas interesadas en continuar con líneas de desarrollo similares pueden emplear los principios descritos, los mismos a su vez pueden llegar a ser funcionales para otro tipo de aplicaciones de la industria.

Como mejora del proyecto se puede implementar un sistema de embalaje, empaquetamiento y automatización del movimiento de los planos.

Existen otros materiales que se pueden emplear para la construcción de la máquina selectora, tales como: aceros inoxidables o aluminio para así poder evitar problemas de corrosión en la estructura y demás partes que la componen.

\section{REFERENCIAS}

[1]. ACUÑA ACUÑA, Jorge. Automatización Industrial; definición y conceptos. Tecnología en marcha. Vol $10,1990$.

[2]. AGUDELO TORO, Diego Fernando, TABARES VALENCIA, Juan Pablo. Diseño de una maquina clasificadora de tuercas automatizada. Trabajo de grado para optar al título de Ingeniero en Mecatrónica. Universidad Tecnológica de Pereira. Pereira 2011.

[3]. GROOVER, M.P. (1987). Automation, Production Systems and Integrated Manufacturing. Prentice Hall.

[4]. H. Meixner, E. Sauer. Introducción a la electroneumatica. Edición 04/90
[5]. H. Meixner, E. Sauer. Iniciación a la electroneumatica, manual de estudio para el seminario FESTO PE 23.

[6]. H. Meixner, Kobler. Introducción a la neumática. Festo-Didactic, Esslinger.

[7]. JONES, Franklin D. Ingenious Mechanisms For Designers and Inventors Volume 1. Estados Unidos: Industrial Press Inc. 1951.

[8]. JONES, Franklin D. Ingenious Mechanisms For Designers and Inventors Volume 3. Estados Unidos: Industrial Press Inc. 1951.

[9]. JONES, Franklin D. Ingenious Mechanisms For Designers and Inventors Volume 4. Estados Unidos: Industrial Press Inc. 1951.

[10]. PIEDRAHITA, Ramón. Ingeniería de la automatización industrial. Editorial RA-MA. Segunda Edición, México DF 2004.

[11]. W, Willian. Seto. Teoría y problemas de vibraciones mecánicas. Registrado en 1970. Editorial McGraw-Hill.

[12]. BELLO PEREZ, Rafael. El Diseño de Ingeniería y la Inteligencia Artificial Revista Facultad de Ingeniería.

[13]. HOYOS MESA, sobre nuevos sistemas de manufactura. Ed. 11. Revista Universidad Autónoma de Occidente. Octubre 1995.

[14]. SlideShare. (2010) Análisis de vibraciones. [Publicación en línea]. Disponible: http://es.slideshare.net/RamdolfAntonioArveloLopez /analisis-de-vibraciones

[15]. (2011) Automatización Industrial. Historia de la Automatización Industrial. [Publication en lineal] Disponible: https://automatizacionindustrial.wordpress.com/2011 /02/17/historia-de-la-automatizacion-industrial/

[16]. (2016). Programa de diseño. Capitulo 5 Cargas variables - Teoría de Fatiga. [Publicación en línea]. http://blog.utp.edu.co/lvanegas/dis1/

Disponible:

[17]. Automatización Industrial. Wiki pedía. [Publicación en línea]. Disponible: http://es.wikipedia.org/wiki/Automatizaci\%C3\%B3n _industrial 
[18]. Pereira. Wiki pedía. [Publicación en línea]. Disponible: http://es.wikipedia.org/wiki/Pereira

[19]. Vibraciones UTP. [Publicación en línea]. Disponible:

https://sites.google.com/site/vibracionesutp/

[20]. Historia de la Automatización. [Publicación en línea]: Disponible: http://tesis.uson.mx/digital/tesis/docs/21319/Capitulo 1.pdf

[21]. Neumática Industrial para todos, Ing. Miguel A. Morales G. [Publicación en línea]. Disponible:

https://sites.google.com/site/neumaticaparatodos/actu adores-neumaticos/actuadores-de-giro-y-motores

[22]. RockWell Automation. Sensores de proximidad e interruptores de final de carrera. [Publicación en línea]. Disponible: http://literature.rockwellautomation.com/idc/groups/1 iterature/documents/br/prxlim-br001_-es-p.pdf

[23]. Rock Well Automation. Interruptores de final de carrera. [Publicación en línea]. Disponible: http://ab.rockwellautomation.com/es/SensorsSwitches/Limit-Switches

[24]. Sensor final de carrera. Wiki Pedía. [Publicación en línea]. Disponible: https://es.wikipedia.org/wiki/Sensor_final_de_carrera

[25]. Relé. Wiki Pedía. [Publicación en línea]. Disponible:

https://es.wikipedia.org/wiki/Rel\%C3\%A9

[26]. Relé. [Publicación en línea]. Disponible: http://platea.pntic.mec.es/ pcastela/tecno/documento s/apuntes/rele.pdf

[27]. Válvulas de solenoide. [Publicación en línea]. Disponible:

http://www.imcosamex.com/nueva/wpcontent/uploads/2013/07/funcionan-valvulassolenoides.pdf

[28]. Interruptor. Wiki Pedía. [Publicación en línea]. https://es.wikipedia.org/wiki/Interruptor

[29]. Fenómeno de fatiga, Universidad Carlos III de Madrid. [Publicación en línea]. Disponible: http://ocw.uc3m.es/ingenieria-mecanica/tecnologiade-maquinas/material-de-clase-

1/fatiga_transparencias.pdf
[30]. Simbología en circuitos neumáticos, Universidad de Cantabria. [Publicación en línea]. Disponible:

http://personales.unican.es/renedoc/Trasparencias $\% 2$ 0WEB/Trasp\%20Neu/T21\%20\%20Simbologia.pdf

[31]. Símbolos neumáticos. [Publicación en línea]. Disponible: http://isa.uniovi.es/docencia/ra_marina/cuatrim2/Tem as/simbolosneumaticos.pdf

[32]. Apuntes de neumática FESTO. [Publicación en línea]. Disponible: http://www.dte.us.es/tec_ind/electron/ai/descarga/Ap untes\%20De\%20Neumatica\%20FESTO.pdf

[33]. Actuadores giratorios DSR/DSRL. [Publicación en línea]. Disponible: https://www.festo.com/cat/esco_co/data/doc_es/PDF/ES/DSR_ES.PDF 